\title{
Fast Handoff Scheme for Seamless Multimedia Service in Wireless LAN
}

\author{
Hye-Soo Kim, Sang-Hee Park, Chun-Su Park, \\ Jae-Won Kim, and Sung-Jea Ko \\ Department of Electronics Engineering, Korea University, \\ Anam-Dong Sungbuk-Ku, Seoul, Korea \\ \{hyesoo, jerry, cspark, jw9557, sjko\}@dali.korea.ac.kr
}

\begin{abstract}
In this pape1 1 , we propose a fast handoff method for seamless multimedia service in IEEE 802.11 WLAN. The proposed method uses an improved access point (AP) with additional radio frequency (RF) module, SNIFFER, monitoring the movement of the station (STA). By using the SNIFFER, the proposed method can completely remove the probe delay. Furthermore, we also propose an effective handoff decision method taking into account the quality of service (QoS) in the application layer. The proposed method uses packet loss information and the received signal strength indication (RSSI) for the handoff decision. Experimental results show that the proposed method can improve the performance of the seamless multimedia service by drastically reducing the handoff delay and packet loss.
\end{abstract}

\section{Introduction}

The main issue of WLAN is to provide seamless host mobility during the handoff that is the mechanism occurred when an STA moves its association from one AP to another [1-8]. During a handoff, no frames should be lost, and the data stream to the video client should be kept as smooth as possible. Hence a fast and efficient handoff scheme is needed for real-time multimedia service to maintain connectivity, minimize data loss and latency while crossing cell boundaries during data transfers [2]-[3].

The handoff procedure in the WLAN can be divided into three distinct logical phases: probing, authentication, and reassociation. In the probing phase, an STA scans for APs by sending ProbeRequest messages (Active Scanning) or listening for Beacon messages (Passive Scanning). After scanning all channels, an AP is selected by the STA using the RSSI. The STA exchanges IEEE 802.11 authentication messages with the selected AP. Finally, if the AP authenticates the STA, an association moves from an old AP to a new AP by exchanging reassociation messages.

The major problem of handoff procedure in WLAN is the handoff delay introduced when an STA is unable to communicate with a certain AP or when

\footnotetext{
${ }^{1}$ This work is supported by a grant from Samsung Advanced Institute of Technology.
} 
the user mobility increases in the WLAN. The handoff delay in the WLAN is divided into three types; probe delay, authentication delay, and reassociation delay. It is well known that the probe delay is the primary contributor to the overall handoff latency [4. Thus, the probe delay has to be significantly reduced for the seamless multimedia communications services.

In this paper, we propose a fast handoff method for seamless multimedia service in IEEE 802.11 WLAN. The proposed method uses a modified AP with SNIFFER which is the additional RF module monitoring the movement of the STA. In the conventional handoff method, an STA has to scan all channels to detect the most probable AP in the probing phase. On the other hand, the proposed method can completely remove the probing phase, i.e. zero probing delay, since the STA can obtain the information about the adjacent AP by using the SNIFFER module. Moreover, the AP with dual RF module can provide the network information to the STA by using the modified neighbor graph (NG) which represents the set of potential next APs.

We also propose an effective handoff decision method taking into account the QoS in the application layer. In general, the handoff occurs when the STA can not detect enough signal strength from the AP. This handoff method, however, may be inefficient since serious packet losses are often generated even when the measured signal is strong enough. In the proposed handoff method, we use packet loss information for the handoff initiation and the variance of the RSSI for the handoff decision.

This paper is organized as follows. In Section 2, we briefly review the IEEE 802.11, conventional handoff procedure, and NG. In Section 3, the proposed fast handoff method is introduced in detail. The experimental results are shown in Section 4 and we give some concluding remarks in Section 5

\section{Backgrounds}

Before introducing the proposed methods, we briefly review the IEEE 802.11 and the concept of the NG, which are the base of the proposed methods.

\subsection{IEEE 802.11 Overview}

The IEEE 802.11 standard uses a basic service set (BSS) for the basic building block of networks. An STA is free to move within the BSS, but it can no longer communicate directly with other stations if it leaves the BSS. An independent BSS (IBSS) is a standalone BSS that has no backbone infrastructure and consists of at least two wireless stations as shown in Fig. 1 (a). This type of network is often referred to as ad-hoc network because it can be constructed quickly without much planning. The ad-hoc wireless network will satisfy most needs of users occupying a smaller area, such as a single room, sales floor, or hospital wing.

For requirements exceeding the range limitations of the independent BSS, the 802.11 standard defines an extended service set (ESS), which is identified by its service set identifier (SSID), as illustrated in Fig. 1 (b). This type of 


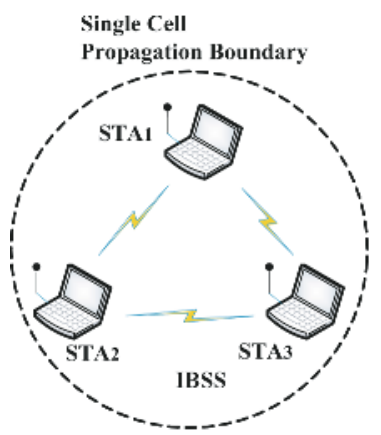

(a)

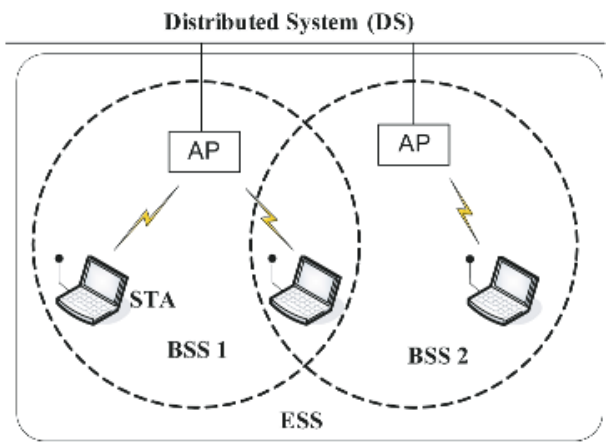

(b)

Fig. 1. The IEEE 802.11 topology. (a) Ad-hoc network, (b) Infrastructure network.

configuration satisfies the needs of large coverage networks of arbitrary size and complexity. An ESS of 802.11 WLAN consists of multiple cells interconnected by APs and a distributed system (DS), such as ethernet [1]-[5]. The STA can change the BSS where it is to be connected, using the active/passive scanning and reassociation service. In fact, while an STA is associated with a BSS, it can decide that the connection quality is poor, so it scans the medium to search for a more reliable connection. If the search is successful, it can decide to invoke the reassociation request to a new AP. If the reassociation response is successful, the STA has roamed to the new AP. Normally, an old AP is notified through the DS. Otherwise, if the reassociation request fails, the STA tries to search for a new BSS [6].

\subsection{Conventional Handoff Procedure in 802.11 WLAN}

A handoff occurs when an STA moves beyond the radio range of one AP, and enters another BSS (at the MAC layer). An STA continuously monitors the signal strength and link quality from the associated AP. If the signal strength is too low, the STA scans all the channels to find a neighboring AP that produces a stronger signal. By switching to another AP referred to as handoff. During the handoff, management frames are exchanged between the STA and the AP. Fig. 2 shows the sequence of messages typically observed during a handoff process. The handoff process starts with the first probe request message and ends with a reassociation response message from an $\mathrm{AP}$.

The complete handoff procedure can be divided into three distinct logical phases: scanning, authentication, and reassociation. During the first phase, an STA scans for APs by either sending ProbeRequest messages (Active Scanning) or listening for Beacon messages (Passive Scanning). After scanning all the channels, the STA selects an AP using the RSSI, link quality, and etc. The STA exchanges IEEE 802.11 authentication messages with the selected AP. Finally, if the AP authenticates the STA, an association moves from an old AP to a new AP as following steps: 


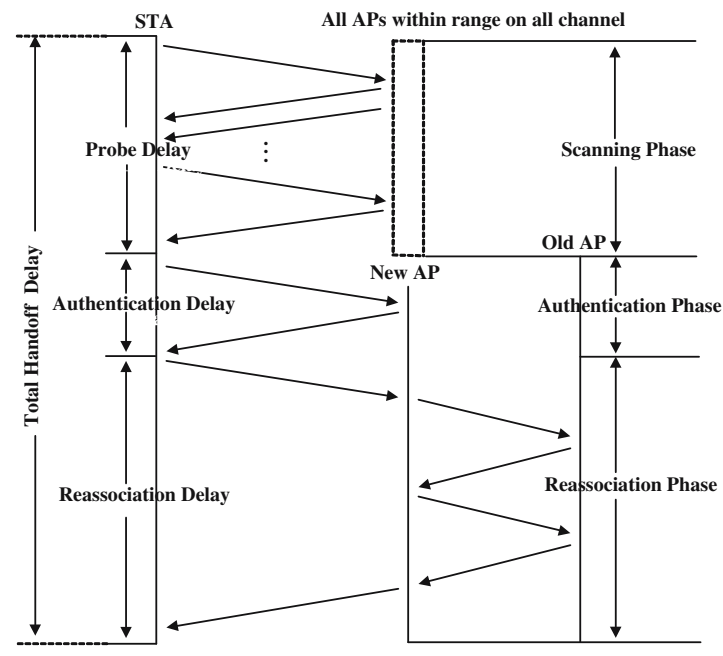

Fig. 2. IEEE 802.11 handoff procedure with IAPP

(1) An STA issues a ReassociationRequest message to a new AP. The new AP must communicate with the old AP to confirm that a previous association existed;

(2) The new AP processes the ReassociationRequest;

(3) The new AP contacts the old AP to finish the reassociation procedure with IAPP [?];

(4) The old AP sends any buffered frames for the STA to the new AP;

(5) The new AP begins processing frames for the STA.

The delay incurred during these three phases is referred to as the link layer (L2) handoff latency, that consists of probe delay, authentication delay, and reassociation delay. Mishra [4] showed that scanning delay is dominant among three delay. Therefore, to solve the problem of L2 handoff delay, scanning delay has to be reduced.

\subsection{Concept of the NG}

In this subsection, we describe the notion and motivation for the NG, and the abstractions they provide. Given a wireless network, the NG containing the reassociation relationship is constructed. Fig. 3 (a) and (b) show the physical topology of the wireless network and the corresponding NG [7].

Reassociation Relationship: Two APs, $a p_{i}$ and $a p_{j}$, are said to have a reassociation relationship if it is possible for an STA to perform an IEEE 802.11 reassociation through some path of motion between the physical locations of $a p_{i}$ and $a p_{j}$. The reassociation relationship depends on the placement of APs, signal strength, and other topological factors and in most cases corresponds to the physical distance (vicinity) between the APs. 


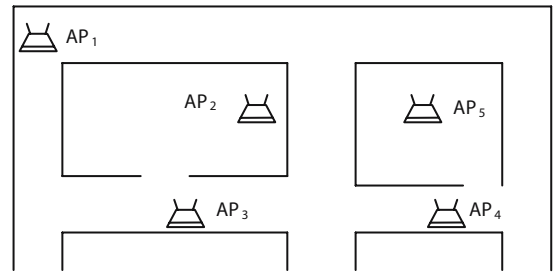

(a)

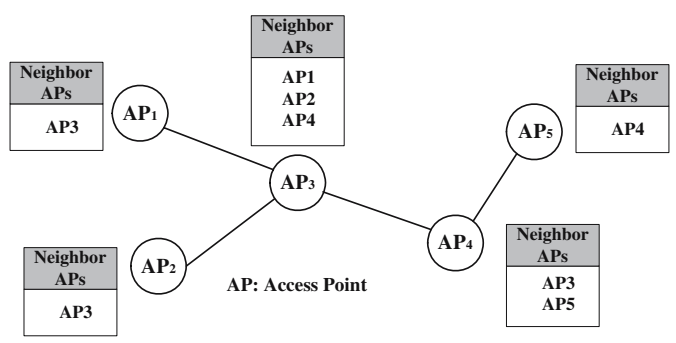

(b)

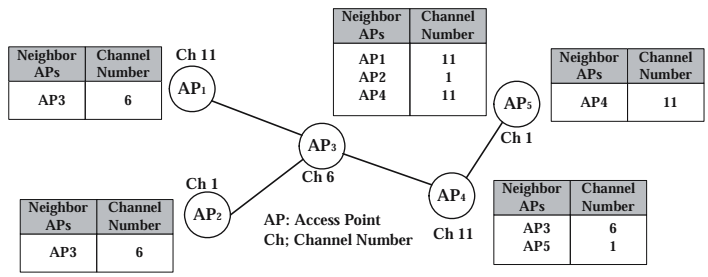

(c)

Fig. 3. Concept of the NG. (a) Placement of APs, (b) Conventional NG, (c) Modified NG

Data Structure (NG): Define an undirected graph $G=(V, E)$ where $V=$ $\left\{a p_{1}, a p_{2}, \cdots, a p_{n}\right\}$ is the set of all APs constituting the wireless network. And the set $E$ includes all existing edges $e_{i j}$ 's where $e_{i j}=\left(a p_{i} ; a p_{j}\right)$ represents the reassociation relationship. There is an edge $e_{i j}$ between $a p_{i}$ and $a p_{j}$ if they have a reassociation relationship. Define $N\left(a p_{i}\right)=\left\{a p_{i_{k}}: a p_{i_{k}} \in V, e_{i k} \in E\right\}$, i.e., the set of all neighbors of $a p_{i}$ in $G$.

The NG can be implemented either in a centralized or a distributed manner. In this paper, the NG is implemented in a centralized fashion, with correspondent node $(\mathrm{CN})$ storing all the NG data structure. The NG can be automatically generated by the following algorithm with the management message of IEEE 802.11.

(1) If an STA associated with $A P_{j}$ sends Reassociate Request to $A P_{i}$, then add an element to both $N\left(a p_{i}\right)$ and $N\left(a p_{j}\right)$ (i.e. an entry in $A P_{i}$, for $j$ and vice versa); 
(2) If $e_{i j}$ is not included in $E$, then create new edge. The creation of a new edge requires longer time and can be regard as 'high latency handoff'. This occurs only once per edge.

The NG proposed in 7] uses the topological information on APs. Our proposed algorithm, however, requires channels of APs as well as topological information. Thus, we modify the data structure of NG as follows:

$$
\begin{aligned}
& G^{\prime}=\left(V^{\prime}, E\right), \\
& V^{\prime}=\left\{v_{i}: v_{i}=\left(a p_{i}, \text { channel }\right), v_{i} \in V\right\}, \\
& e_{i j}=\left(a p_{i}, a p_{j}\right), \\
& N\left(a p_{i}\right)=\left\{a p_{i_{k}}: a p_{i_{k}} \in V^{\prime}, e_{i k} \in E\right\},
\end{aligned}
$$

where $G^{\prime}$ is the modified NG, and $V^{\prime}$ is the set which consists of APs and their channels. Therefore, we add the channel index to the conventional NG as shown in Fig. 3 (c). In Section 3, we develop a fast handoff algorithm based on the modified NG.

\section{Proposed Fast Handoff Method}

In this section, we present a fast handoff method with the AP with dual RF modules by using the modified NG described in Section 2 .

\subsection{Proposed AP with Dual RF Modules}

In IEEE 802.11 WLAN, a handoff is controlled by the STA [9]-[10]. Before the handoff to the new AP, an STA continuously monitors the signal strength and link quality of the associated AP. If the signal strength is lower than a certain threshold, the STA scans all channels to find an AP that produces the strongest signal. This procedure is known as the probing (or scanning). It is known that the probe delay is a dominant part of the entire handoff delay.

To remove the probe delay, we propose a new type of handoff method in WLAN. As shown in Fig. 4(a), the conventional AP contains a single RF module that can receive and transmit signals by turns in the allotted channel.

Since the conventional AP uses only one channel, it can not detect the movement of STA communicating with another AP. For example, as shown in Fig. [4 (c), the AP1 can not detect the STA3 associated with AP2 even when the STA3 enters the BSS of the AP1. Therefore, we add an additional RF module, SNIFFER, to monitor the channels of adjacent APs as shown in Fig. 4(b). By using the SNIFFER, the proposed AP can eavesdrop the medium access control (MAC) frame of incoming STA3 as shown in Fig. 4(d). By examining MAC frame received from the STA3, the AP1 can obtain the address of AP2 associated with STA3. Then, the information on AP1 is transferred to the STA3 through AP2. Since the proposed AP can provide the scanning results to the STA, the probe delay at the STA is eliminated. 


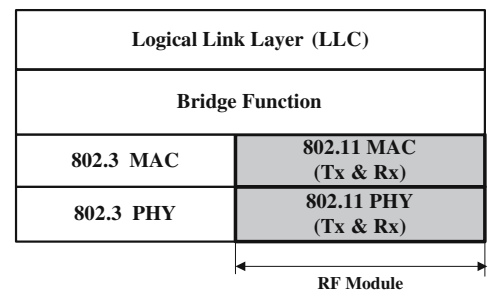

(a)

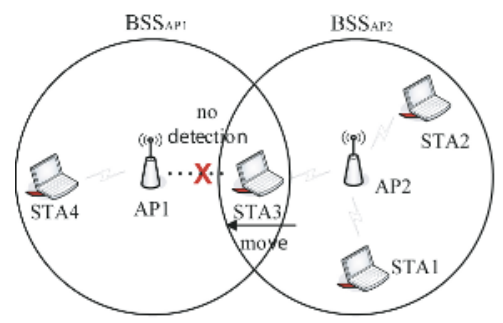

(c)

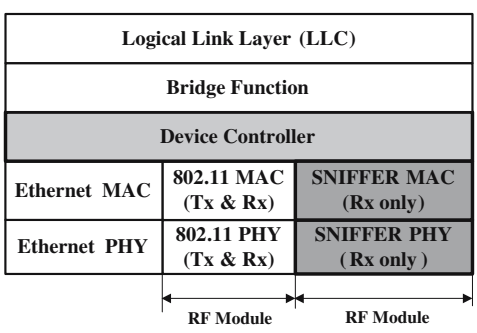

(b)

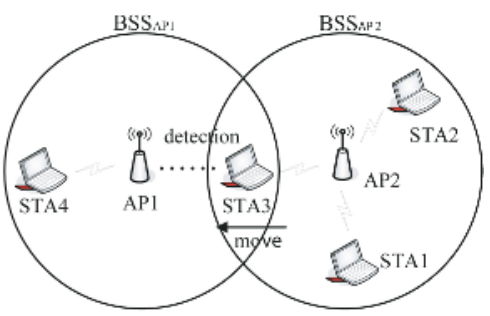

(d)

Fig. 4. The architecture of APs. (a) Conventional AP with single RF module, (b) Proposed AP with dual RF modules, (c) Example using conventional AP, (d) Example using proposed AP.

\subsection{Proposed Handoff Decision Method}

Traditional handoff decision algorithms are based on the RSSI [11-[12]. If the RSSI is smaller than a certain threshold, the handoff from one AP to the other occurs. However, this handoff decision method has some drawbacks that serious packet losses can be generated even when the RSSI from the associated AP is strong enough. Moreover, a big fluctuation in RSSI causes handoff oscillation, i.e. ping-pong phenomenon, so that the STA wanders between two cells and the quantity of the packet loss increases significantly.

In this subsection, we propose an effective handoff decision method using packet loss ratio (PLR) for the L2 handoff. Fig. 6] shows the relationship between the PLR and RSSI.

As an STA moves away from its current AP, the RSSI measured at the AP decreases, while the PLR increases. In the conventional handoff decision method, the STA does not start the handoff procedure until the RSSI value is lower than a certain threshold. If the threshold is not sufficiently high, serious packet losses can be introduced in the conventional method. On the other hand, the proposed decision method starts the handoff initiation step if the PLR at the STA is greater than a certain threshold. Therefore, the proposed decision method can reduce the quality degradation introduced by the handoff.

In the handoff initiation step, the STA monitors the variations of the RSSI from the current AP and receives that from the SNIFFER of the new AP. Since the RSSI fluctuates over time, we use the median filter with length $2 k+1$ defined as: 


$$
Z_{i}(x)=\operatorname{med}\left(R_{i}(x-k), \ldots, R_{i}(x), \ldots, R_{i}(x+k)\right),
$$

where $x$ is the position of the STA and $R_{i}(x)$ is the RSSI value from the $A P_{i}$. As the STA becomes closer to the AP2, the median of RSSIs from the AP2 increases, whereas that from the current AP decreases. Since $Z_{i}(x)$ reflects the direction of the STA and signal strength of the APs, we define the handoff decision parameter, $H(x)$, which is given by

$$
H(x)=Z_{i}(x)-Z_{j}(x),
$$

where $Z_{i}(x)$ and $Z_{j}(x)$, respectively, are the median values of RSSI from $A P_{i}$ and $A P_{j}$. If $H(x)$ is smaller than a certain threshold $T$, the handoff to the new AP is performed. Otherwise, the association with the current AP is maintained.

\subsection{Fast Handoff Procedure}

The proposed handoff method is performed in both the L2. Fig. 5 illustrates a flow of the proposed handoff method. The AP1 and AP2, respectively, have the communication channels 1 and 6 . As the STA moves toward AP2 from AP1, the handoff procedure is performed as follows:

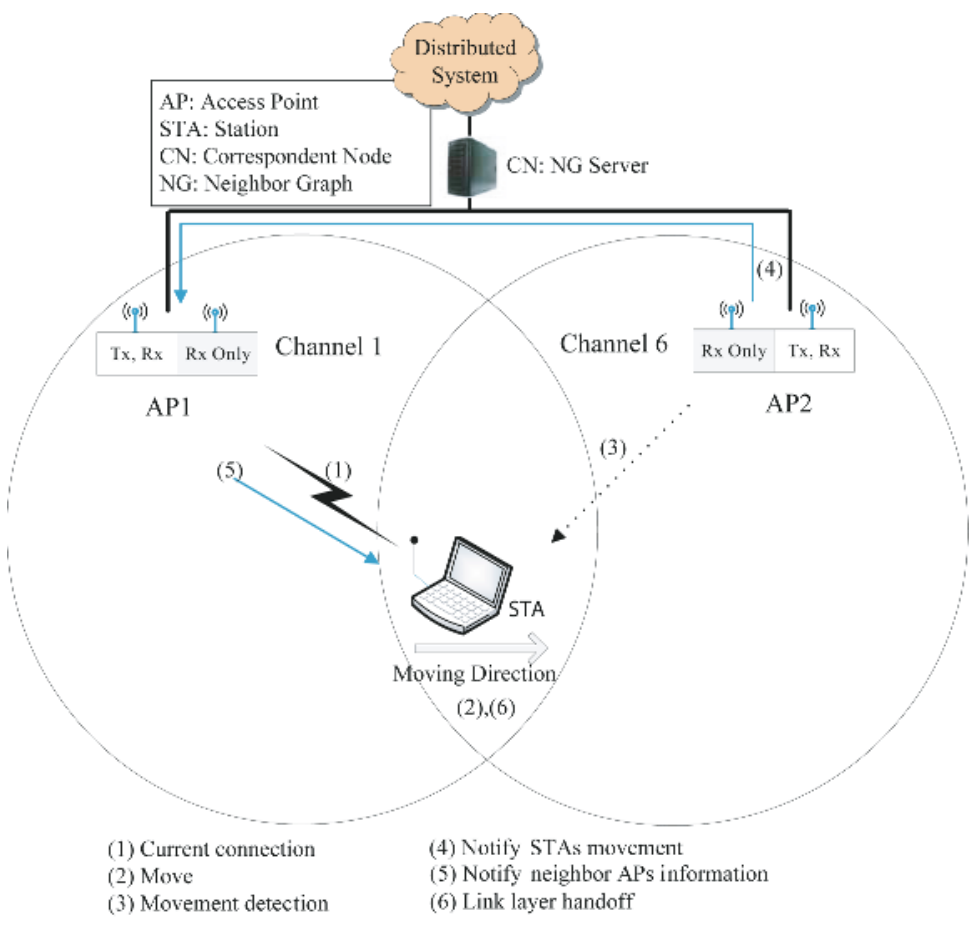

Fig. 5. Procedure of the proposed fast handoff method 


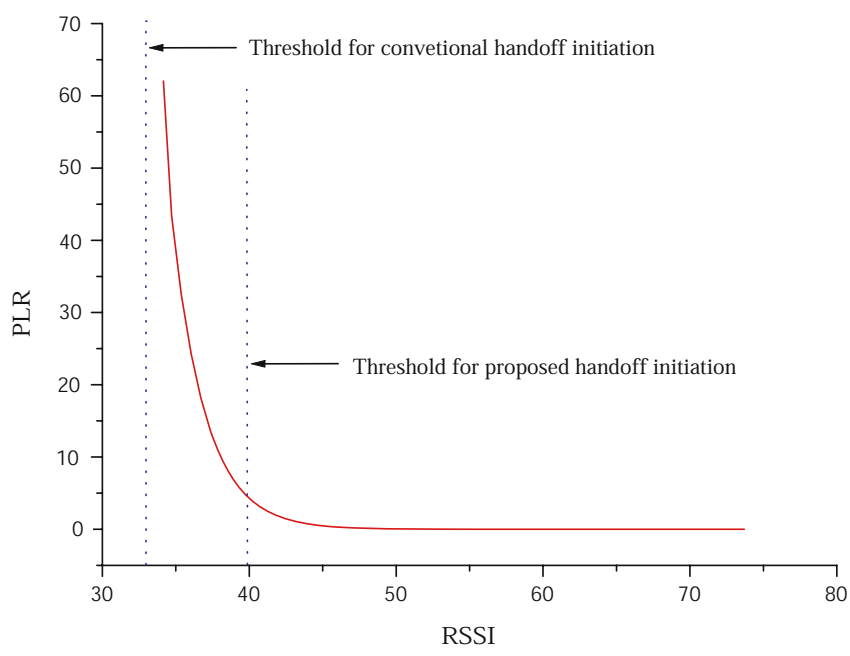

Fig. 6. The relation between the PLR and RSSI

(1), (2) The STA associated with AP1 moves toward AP2;

(3) The SNIFFER module of AP2 receives the MAC frames of the STA;

(4) Using the destination address in the MAC frame of the STA, the SNIFFER of AP2 can be aware of information on the AP1. Then, the information is saved in the NG and transferred to AP1;

(5) By investigating the $\mathrm{NG}$, the AP1 relays the channel information on the AP2 to the STA;

(6) The STA monitors whether packet losses occur or not. If the packet losses are detected, the L2 handoff is initiated. Then, the L2 handoff is performed when $H(x)>T$;

As described in Section 3.1, the proposed handoff algorithm can eliminate the probe delay by using the SNIFFER module added to AP. And the STA can be authenticated and associated before handoff by using the NG. Therefore, the proposed handoff method can drastically reduce the L2 handoff delay.

\section{Experimental Results}

We developed an experimental platform in order to evaluate the performance of the proposed method. To exchange the NG information, the socket interface is used. The device driver of a common WNIC was modified so that the STA operates as an AP that can support the handoff initiation message. We have developed NG Server, NG Client, and SNIFFER for the proposed mechanism. The $N G$ Server manages the data structure of NG on the experimental platform and processes the request of the $N G$ Client that updates the NG information on the STA after the STA moves to the another AP. Using destination address in 
the MAC frame of the STA, the SNIFFER can be aware of the AP associated with the STA. If the AP can support the requirement of the STA, the SNIFFER sends the old AP a message including handoff information such as the measured RSSI, the available throughput, the MAC address of the STA, and so on.

Fig. 7 shows the median values of RSSIs, $Z_{1}(x)$ and $Z_{2}(x)$. As the STA becomes closer to the AP2, $Z_{2}(x)$ increases, whereas $Z_{1}(x)$ decreases. To evaluate the performance of the proposed method in terms of handoff delay, we

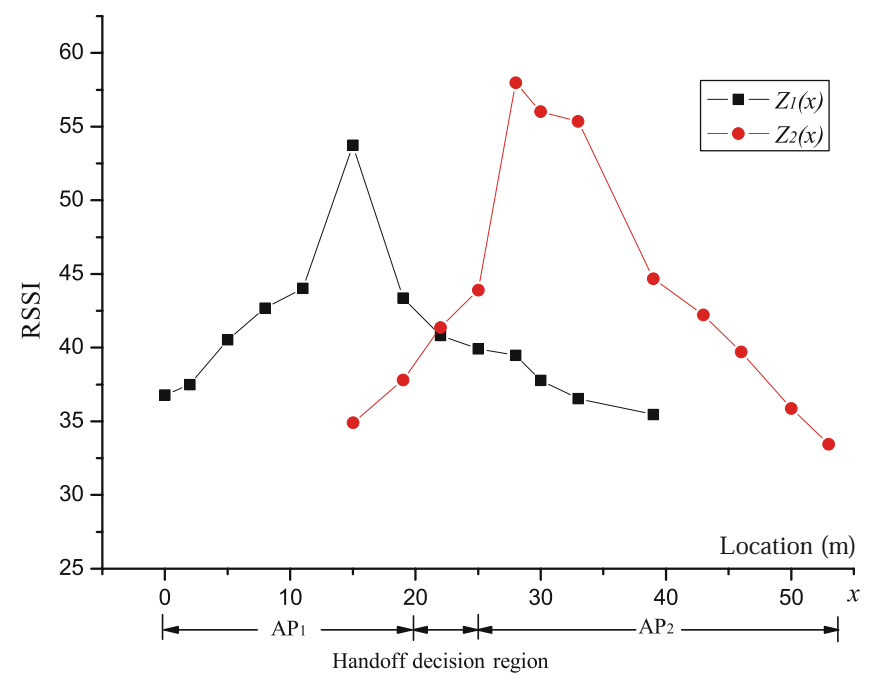

Fig. 7. Median values of RSSI

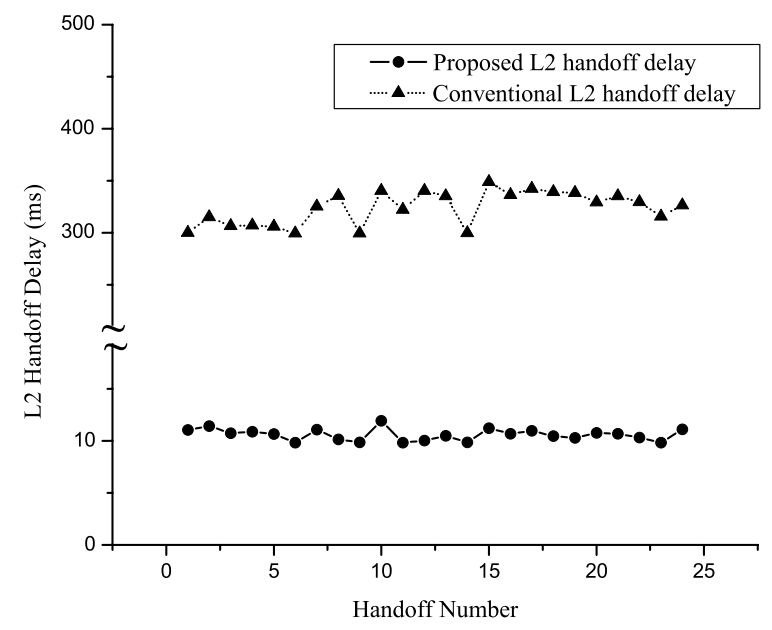

Fig. 8. L2 handoff delay 


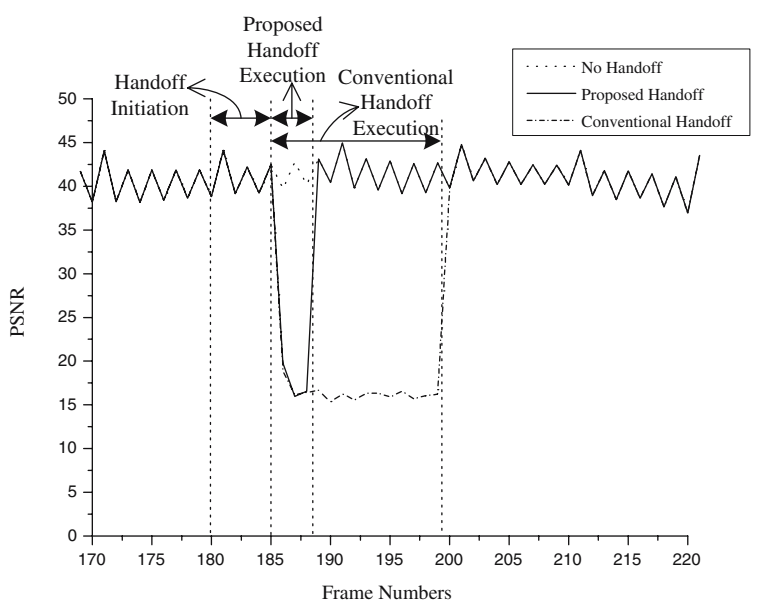

Fig. 9. PSNR during handoff

measured the handoff delay at the L2. As shown in Fig. 8, the value of average L2 handoff delay incurred by the proposed method is much lower than that of the conventional L2 handoff delay [4].

The test sequence of Akiyo in QCIF format $(176 \times 144)$ is used to examine the performance of the proposed handoff decision method. The sequence with 300 frames is transmitted to the client at the rate of 30 frames per second. We developed the video streaming server so as to show that the proposed method can improve the QoS during handoff.

Fig. 9] shows the peak signal-to-noise ratio (PSNR) results of the transmitted streaming video when the handoff occurs at the 185 frame. The quality of video in the conventional handoff decision method is significantly degraded just before handoff since there are a lot of packet losses before the handoff initiation. On the other hand, the proposed method can provide seamless video streaming during handoff procedure, since the handoff is initiated before the PLR becomes too low.

\section{Conclusions}

In this paper, we have presented a fast handoff method for seamless multimedia service using the AP with dual RF modules. Since the SNIFFER module monitors the movement of the STA, the proposed method can remove the probe delay which is the dominant part among the three types of L2 handoff delays. We also have proposed an effective handoff decision method using the PLR and the RSSI. By determining the optimal time to handoff, the proposed method improves the QoS during handoff. Experimental results indicate that seamless multimedia service can be achieved with the proposed method in WLAN. 


\section{References}

1. Gast, M. S.: 802.11 Wireless Networks. O'REILLY (2002) 1-150

2. ISO/IEC 8802-11 - ANSI/IEEE Std 802.11: Information Technology Part 11: Wireless Lan Medium Access Control (MAC) and Physical Layer (PHY) Specifications. IEEE (1999)

3. Cheng, L. T., Pink, S., Lye, K., M.: A fast handoff scheme for wireless networks. Proceeding of the 2nd ACM international workshop on wireless mobile Multimedia (1999) 83-90

4. Mishra, A., Shin, M., H., Albaugh, W.: An Empirical Analysis of the IEEE 802.11 MAC Layer Handoff Process. ACM SIGCOMM Computer Communication Review 3 (2003) 93-102

5. Geier, J.: Wireless LANs, Second Edition. SAMS 2 (2001)

6. Ramjee, P., Luis, M.: Wireless LANs and WPANs towards 4G wireless. Artech House (2003)

7. Mishra, A., Shin, M., H., Albaugh, W.: Context Caching using Neighbor Graphs for Fast Handoff in a Wireless Network. Computer Science Technical Report CSTR-4477 (2003)

8. Balachandran, A., Voelke, G., Bahl, P., Rangan, P.: Characterizing User Behavior and Network Performance in a Public Wireless LAN. Proceeding of ACM SIGEMTRICS (2002)

9. Caceres, R., Padmanabhan, V., N.: Fast and Scalable Wireless Handoffs in Support of Mobile Internet Audio. Moblie Networks and Application 3 (1998) 180-188

10. Akyildiz, I., F.: Mobility management in next-generation wireless systems. Proceedings of the IEEE 87 (1999) 1347-1384

11. Chia, S., Warburton, R.: Handoff Creteria for City Microcellular Radio Systems. Proceeding of VTC (1990) 276-281

12. Chia, S.: The control of Handover Initiation in Microcells. Proceeding of VTC (1991) 531-536 